\title{
Politique
}

Politique

\section{Michel Delapierre et Jean-Benoît Zimmermann, L'informatique du Nord au Sud, Paris, Notes et études documentaires, La documentation française, 1986, 144 p.}

\section{Gérard Boismenu}

Numéro 11, hiver 1987

L’État privé

URI : https://id.erudit.org/iderudit/040563ar

DOI : https://doi.org/10.7202/040563ar

Aller au sommaire du numéro

Éditeur(s)

Société québécoise de science politique

ISSN

0711-608X (imprimé)

1918-6584 (numérique)

Découvrir la revue

Citer ce compte rendu

Boismenu, G. (1987). Compte rendu de [Michel Delapierre et Jean-Benoît Zimmermann, L'informatique du Nord au Sud, Paris, Notes et études documentaires, La documentation française, 1986, 144 p.] Politique, (11), 205-208. https://doi.org/10.7202/040563ar d'utilisation que vous pouvez consulter en ligne.

https://apropos.erudit.org/fr/usagers/politique-dutilisation/ 
Michel Delapierre et Jean-Benoît Zimmermann, L'informatique du Nord au Sud, Paris, Notes et études documentaires, La documentation française, 1986, $144 \mathrm{p}$.

Les auteurs nous présentent une étude de branche. L'informatique, comme un complexe industriel transnationalisé, est saisie dans le faisceau des relations économiques internationales diversifiées auxquelles elle participe. Certaines caractéristiques du profil de la branche au niveau mondial ressortent. On compte un nombre réduit de multinationales qui se livrent une concurrence acharnée, chacune s'appuyant sur le système productif de son pays d'origine et sur la participation de l'État à la formation de cette industrie.

Le rapport "pays d'origine» et "firmes transnationalisées» est l'objet d'une attention particulière de la part des auteurs. Ils soumettent l'hypothèse suivante: «[...] ce n'est pas l'industrie américaine de l'informatique qui domine le monde, mais [...], de par la position des firmes qui en sont originaires, ce sont les États-Unis qui exercent un pouvoir sur le reste du monde à travers le complexe industriel transnationalisé de l'informatique.» (p. 8)

La branche est décrite à travers ses activités, ses caractéristiques technologiques, ses acteurs et la situation mondiale. Il en ressort une homogénéisation des gammes et une standardisation à l'échelle internationale qui est induite par les stratégies de développement des firmes multinationales qui dominent la branche. 
Au-delà de ces caractéristiques d'ensemble, il n'en reste pas moins que l'on connaît une disparité interne à la branche selon que l'on retienne l'un ou l'autre des quatre segments de la production: les ordinateurs universels, les mini-ordinateurs, les micro-ordinateurs et les périphériques. Par exemple, de l'un à l'autre, le degré de concentration (monopolisation) n'est pas homogène et rares sont les entreprises qui interviennent également dans chacun. D'une manière générale, les entreprises couvrent un large éventail de segments, alors que celles qui sont très spécialisées se retrouvent dans le segment des micro-ordinateurs (Apple, Commodore).

L'informatique reste très concentrée dans les pays du Nord. Le parc informatique mondial le montre assez bien: en 1980, bien que ne représentant que $18 \%$ de la population mondiale et $71 \%$ du PNB mondial, les «pays développés» en détiennent $87 \%$ (en valeur). Concurremment, le commerce mondial des produits informatiques est largement circonscrit aux pays industrialisés.

Les trois grands acteurs de ces derniers est nettement inégal. Les États-Unis sont un pays fortement producteur et grand exportateur, vers la Communauté économique européenne et le reste du monde, alors que la Communauté européenne, tout en dépendant des importations en provenance des États-Unis, ses exportations sont destinées principalement à d'autres pays européens. Pour sa part, le Japon consomme peu les produits étrangers et émerge comme un pays fortement exporteur.

Mises à part la position des agents industriels et la place respective des espaces nationaux, tant du Nord que du Sud, face au complexe de l'informatique, les auteurs s'intéressent au rôle de l'État dans la formation des constructeurs nationaux ou comme acheteur international.

Sans aucun doute, le marché du pays d'origine demeure pour la firme multinationale un facteur important de stabilisation de leur position concurrentielle. Cela joue à plus d'un titre: base 
de lancement pour de nouveaux produits, «effet de vitrine» pour les produits, condition pour une politique de prix agressive. Au total, «la bonne marche des opérations domestiques garantit la force de conquête des marchés extérieurs.» (p. 101)

De plus, l'État joue un rôle de premier plan. Aux ÉtatsUnis, les marchés publics et les programmes de R-D se sont avérés deux leviers importants. Au Japon, l'État occupe une place majeure depuis 1956 pour le maintien et le développement d'une industrie informatique nationale. Cependant, l'intervention publique est plus récente en Europe. Elle a consisté à regrouper des forces dispersées et à participer dans le capital de nouveaux groupes; encore une fois, la politique d'achats préférentiels (commandes publiques) et l'aide directe constituent des instruments privilégiés de politique.

Malgré la diversité régionale des pays en voie de développement, il n'en reste pas moins qu'ils sont aux prises avec des problèmes majeurs de coordination $1^{\circ}$ entre les unités de R-D et les unités de production, $2^{\circ}$ dans l'organisation de l'informatisation du secteur public; $3^{\circ}$ dans le processus de planification et de prise de décision. À tout prendre, avec une base industrielle déficiente et un marché domestique restreint, les pays en voie de développement se consacrent davantage à la production de logiciels (surtout les petits pays d'Asie) et à la mise en place d'une formation adaptée afin que les techniciens nationaux soient en mesure de développer des applications appropriées aux besoins des pays de la région.

Au total, ce livre présente une mise en forme d'informations touchant le complexe transnationalisé de l'informatique. Il s'agit en quelque sorte d'une étude de cas qui illustre la problématique élaborée par Charles-Albert Michalet. L'ensemble de l'information est traité d'abord sur le plan descriptif. Si nous possédons globalement les données du problème, les développements, généralement assez succincts, ne nous permettent pas d'approfondir tel ou tel aspect. 
Par exemple, sur la question du rôle de l'État, nous devons nous contenter de quelques notes, somme toute, générales. Soulignant au départ l'existence de deux logiques de nature indépendante, ayant pour agents les États et les firmes, qui fait en sorte que le processus décisionnel est travaillé nécessairement par une "stratégie double», les auteurs concluent, au terme de leur étude, que l'on peut constater des points de friction entre multinationales et États nations résultant de la multiplicité des objectifs et des situations, mais que, dans l'ensemble, les "politiques observables» correspondent «à des stratégies doubles, prenant en compte les deux logiques. » (p. 140) La boucle est bouclée, mais, entre les deux affirmations, le traitement analytique des politiques observables reste assez mince.

À titre de document d'information, ce livre peut être très utile. Il s'ajoute aux diverses études de branche qui ont été réalisées ces dernières années. Sur le plan de la comparaison des branches, on peut regretter que dans cette étude, les auteurs n'approfondissent pas la question des transferts de technologie.

Gérard Boismenu

Université de Montréal 